\title{
BMJ Open Inflammation and nutrition-based biomarkers in the prognosis of oesophageal cancer: a systematic review and meta-analysis
}

\author{
Yan Jiang (D) , ${ }^{1}$ Dian Xu, ${ }^{1,2}$ Huan Song, ${ }^{1}$ Beibei Qiu (D) , ${ }^{1}$ Dan Tian, ${ }^{1}$ Zhongqi Li, ${ }^{1}$ \\ Ye Ji, ${ }^{1}$ Jianming Wang (i) ${ }^{1}$
}

To cite: Jiang $\mathrm{Y}, \mathrm{Xu} \mathrm{D}$, Song $\mathrm{H}$, et al. Inflammation and nutrition-based biomarkers in the prognosis of oesophageal cancer: a systematic review and meta-analysis. BMJ Open 2021;11:e048324. doi:10.1136/ bmjopen-2020-048324

- Prepublication history and additional supplemental material for this paper are available online. To view these files, please visit the journal online (http://dx.doi.org/10.1136/ bmjopen-2020-048324)

YJ, DX and HS contributed equally.

Received 27 December 2020 Accepted 01 September 2021

Check for updates

(c) Author(s) (or their employer(s)) 2021. Re-use permitted under CC BY-NC. No commercial re-use. See rights and permissions. Published by BMJ.

${ }^{1}$ Department of Epidemiology, Nanjing Medical University,

Nanjing, China

${ }^{2}$ Department of Epidemiology, Ningbo Municipal Center for Disease Control and Prevention, Ningbo, China

Correspondence to Professor Jianming Wang; jmwang@njmu.edu.cn

\section{ABSTRACT}

Background Accumulating literature has shown the predictive values of inflammation and nutrition-based biomarkers in the prognosis of oesophageal cancer but with inconsistent findings.

Method We performed a meta-analysis to systematically evaluate the predictive value of the neutrophil-tolymphocyte ratio (NLR), platelet-to-lymphocyte ratio (PLR), lymphocyte-to-monocyte ratio (LMR), C reactive proteinto-albumin ratio (CAR), systemic inflammation index (SII), prognostic nutritional index (PNI), Glasgow Prognostic Score (GPS) and modified Glasgow Prognostic Score (mGPS) in oesophageal cancer. The outcome indicators include the overall survival (OS), disease-free survival (DFS) and cancer-specific survival (CSS). We applied pooled HR, sensitivity, specificity, positive likelihood ratio, negative likelihood ratio, diagnostic odds ratio and area under the curve together with $95 \% \mathrm{Cl}$ to estimate the predictive accuracy.

Results A total of 72 studies, including 22260 patients, were included in the meta-analysis. Elevated NLR, PLR CAR, SII, GPS, mGPS and decreased LMR and PNI were associated with poor OS of oesophageal cancer. A high level of NLR, PLR and GPS was related to poor DFS. A high level of NLR and GPS was related to poor CSS. The summarised AUC of CAR $(0.72,95 \% \mathrm{Cl}$ : 0.68 to 0.75$)$ and mGPS $(0.75,95 \% \mathrm{Cl}: 0.71$ to 0.78$)$ surpassed any other indicators.

Conclusions Clinical indicators such as NLR, PLR, LMR, PNI, SII, CAR, GPS and mGPS have the moderate predictive ability in OS, DFS and CSS of oesophageal cancer. The pretreatment level of CAR and mGPS showed an outstanding prediction value in 5-year OS for oesophageal cancer.

\section{BACKGROUNDS}

Globally, oesophageal cancer is the seventh most common cancer and the sixth leading cause of cancer death. ${ }^{1}$ In 2020 , there were 570000 new cases of oesophageal cancer and about 500000 deaths worldwide. ${ }^{2}$ Pathologically, squamous cell carcinoma (SCC) and adenocarcinoma are the major histological types. Oesophageal adenocarcinoma is

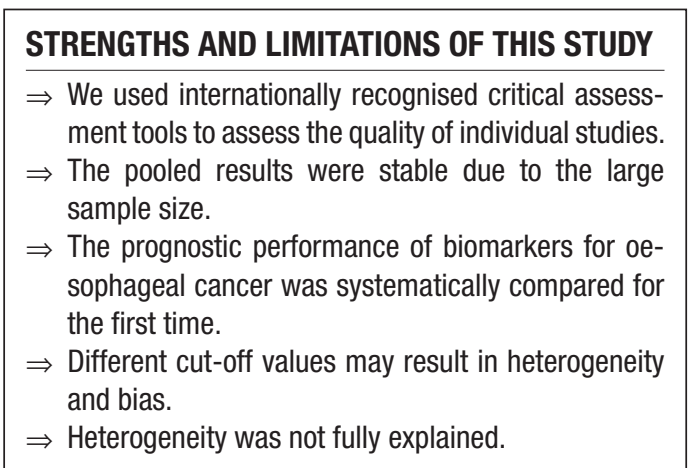

mainly observed in industrialised countries, and nearly half of the cases occur in Northwest Europe and North America, while oesophageal squamous cell carcinoma (ESCC) is more common in China, Central Asia or South Africa. Despite substantial efforts in diagnosis, accurate staging and advanced treatments, the 5-year survival rate remains unfavourable with frequent metastasis and recurrence. ${ }^{3}$ The pathological tumor-nodemetastasis (TNM) stage is the gold standard for predicting oncological outcomes after surgery. ${ }^{4}$ However, with the diversification of treatment methods and the complexity of prognostic factors, prognosis prediction tends to be unsatisfactory. Therefore, it is urgent to find better prognostic biomarkers to guide clinical treatment and appropriate follow-up.

Increasing evidence indicates that systemic inflammatory response and nutritional status are involved in tumour development and influence the clinical prognosis. Principal inflammation-based prognostic scores $^{5-7}$ include a neutrophil-to-lymphocyte ratio (NLR), platelet-to-lymphocyte ratio (PLR), lymphocyte-to-monocyte ratio (LMR), C reactive protein-to-albumin ratio (CAR), systemic inflammation index (SII), pretreatment 
albumin levels and lymphocyte to monocyte ratio. Typical nutrition-based prognostic scores ${ }^{89}$ are prognostic nutritional index (PNI) based on serum albumin and total lymphocyte count, Glasgow Prognostic Score (GPS) based on elevated $\mathrm{C}$ reactive protein (CRP) concentration and low levels of albumin and modified Glasgow Prognostic Score (mGPS), a modified version of GPS. Recently, accumulating literature has shown the prognostic values of these inflammation and nutrition-based prognostic markers in oesophageal cancer, but with inconsistent findings. Hence, it is meaningful to distinguish an accurate prognosis index for patients with oesophageal cancer to guide individualised therapy and precision service.

In the current study, we performed a systematic review of relevant literature. We applied the meta-analysis to explore the accuracy of inflammation and nutrition-based prognostic scores for patients with oesophageal cancer.

\section{MATERIALS AND METHODS \\ Literature search}

We followed the Preferred Reporting Items for Systematic Reviews and Meta-Analyses statement. Two students (YJ and DX) independently searched PubMed, Web of Science and Cochrane Library Databases for eligible articles from the inception of databases to March 2020. Additionally, references in the eligible publications were also reviewed for potential studies. The language was limited to English. The search terms are listed in online additional file 1 . The detailed search procedure is illustrated in figure 1 .

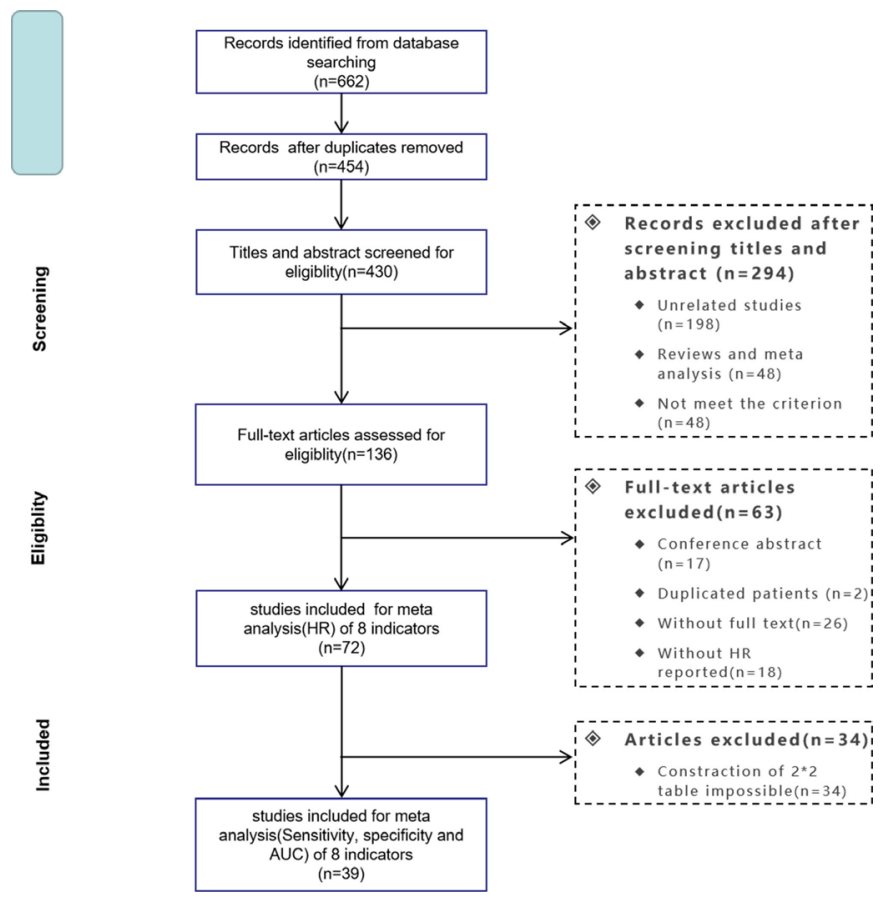

Figure 1 Flow diagram of the search process. AUC, area under the curve

\section{The definition of outcome}

Overall survival (OS) was defined as the time from the beginning of treatment to the death due to all causes or last follow-up. Cancer-specific survival (CSS) was defined as the time from the beginning of therapy to the time of cancer-related death. Disease-free survival (DFS) was defined as the time from the start of treatment to the cancer recurrence or the occurrence of the second primary malignancy. ${ }^{10}$

\section{Selection criteria}

Articles were included if they met the following criteria: (1) patients were histopathologically confirmed to be primary oesophageal cancer; (2) prognostic indicators were measured before esophagectomy, chemotherapy or radiotherapy; (3) hazard ratios (HRs) with 95\% CIs were reported in multivariate analysis. Studies were excluded if they were: (1) reviews, case reports, letters or conference abstracts; (2) studies with insufficient data; or (3) duplicate publications.

\section{Data extraction}

For each study, the following information was extracted by two students ( $\mathrm{YJ}$ and $\mathrm{DX})$ : the name of the first author, year of publication, country, study design, pathological type, number of patients, age, sex, end-point, follow-up time, cut-off selection, therapy, tumour stage, cut-off values and HRs (95\% CIs). We further collected the data of true-positive $(\mathrm{TP})$, false-positive $(\mathrm{FP})$, true-negative $(\mathrm{TN})$ and false-negative (FN) for 5-year OS directly provided in the paper or calculated by comparable data (the number of people in the high-risk and low-risk groups according to the cut-off values and the corresponding number of deaths and survivors). If only the area under the curve (AUC) was reported, we contacted the corresponding author for original data. If we could not get a response, we only included this study in the first part of the analysis.

\section{Quality assessment}

Two reviewers (HS and $\mathrm{BQ}$ ) independently assessed the methodological quality of the studies using the Quality Assessment of Diagnostic Accuracy Studies 2 tool. ${ }^{11}$ Each item was judged as 'yes', 'no' or 'unclear'. Any signalling question answered 'yes' indicated a low risk of bias, while 'no' showed a high risk of bias. If the answer was uncertain, the domain was judged as having an uncertain risk of bias.

\section{Statistical analysis}

The risk of bias was analysed and plotted using Review Manager V.5.3 (London, UK). The meta-analysis was performed using STATA V.15.0 (Texas, USA). The strength of NLR, PLR, LMR, PNI, SII, CAR, GPS, mGPS in association with OS, CSS and DFS was measured by the combined HRs and their 95\% CIs. Cochran's $Q$ test and Higgins $\mathrm{I}^{2}$ statistics were undertaken to assess the heterogeneity of studies. If $\mathrm{p} \geq 0.10$ in the $\mathrm{Q}$ test or $\mathrm{I}^{2}<50 \%$, we used the fixed-effect model; otherwise, we used the random-effect model. Publication bias was assessed by 
Begg and Egger test. The sensitivity analysis was utilised by omitting individual study one-by-one to evaluate the robustness of the results. All $\mathrm{p}$ values were two-tailed, and a $p$ value $<0.05$ was considered statistically significant.

The pooled sensitivity, specificity, AUC and the corresponding $95 \%$ CI were calculated by TP, FP, FN and TN using a bivariate regression model. The threshold effects were calculated by testing the Spearman correlation using Meta-DiSc (Madrid, Spain). ${ }^{12}$ If $^{2} \geq 50 \%$ and $p$ value $\leq 0.05$, the heterogeneity was significant due to the nonthreshold effect, and then we used the meta-regression analysis to find the source of heterogeneity. The pooled positive likelihood ratio (P-LR), negative likelihood ratio (N-LR) and diagnostic odds ratio (DOR) were also calculated to understand the performance of the prognostic index better. Deek's funnel plot was used to detect publication bias. To evaluate the difference of AUC between biomarkers, we checked the overlap of $95 \%$ CIs. If not, we used the following $z$-test $\left(\left(X_{1}-X_{2}\right) /\left(S E_{1}^{2}+S E_{2}^{2}\right)^{1 / 2}\right)$, where $X_{1}$ and $X_{2}$ represented the indicators, and $S E_{1}$ and $S E_{2}$ were the corresponding standard errors. ${ }^{13}$ It was considered significantly different if the $\mathrm{p}$ value obtained from the z-test was less than $\mathrm{P}^{\prime}(0.05 / n, n$ was the number of comparisons). The comparison for sensitivity, specificity, P-LR, N-LR, or DOR was also performed.

\section{RESULTS}

\section{Literature selection and study characteristics}

The initial search identified 662 potentially relevant records. After removing duplicates and papers that did not meet the inclusion criteria, 72 studies with 22260 subjects remained for the systematic review (online additional file 2). A flowchart demonstrating the process of study selection is illustrated in figure 1 . Most studies were carried out in Asia (42 in China; 23 in Japan). Before treatment, the blood cell counts used to calculate NLR, PLR, LMR and CAR were obtained. The baseline characteristics and treatment methods are presented in online additional file 2 .

\section{Risk-of-bias and quality assessments}

Figure 2 illustrates the risk assessment of bias. A high risk of selection bias was observed in all studies. Nearly one-third of the studies had an unclear bias in study attrition. One study had an unclear bias for detection bias, and two studies had the risk of bias in measuring prognostic factors and outcomes, respectively. Six studies were judged as having an unclear performance bias.

\section{Prognostic indicators in OS, DFS and CSS of esophageal cancer}

As shown in figure $3(\mathrm{~A}-\mathrm{H})$, factors significantly contributing to a short OS were a high level of NLR (HR: 1.43, $95 \%$ CI: 1.30 to $\left.1.58, \mathrm{p}<0.001 ; \mathrm{I}^{2}=61.7 \%, p_{\text {het }}<0.001\right)$, PLR (HR: $1.26,95 \%$ CI: 1.18 to $1.35, \mathrm{p}<0.001 ; \mathrm{I}^{2}=29.8 \%$, $p_{\text {het }}=0.108$ ), CAR (HR: $1.84,95 \%$ CI: 1.60 to $2.10, \mathrm{p}<0.001$; $\mathrm{I}^{2}=41.8 \%, p_{h e t}=0.079$ ), SII (HR: $1.46,95 \%$ CI: 1.30 to 1.65 , $\left.\mathrm{p}<0.001 ; \mathrm{I}^{2}=41.0 \%, p_{h e t_{2}}=0.118\right)$, GPS (HR: $2.35,95 \% \mathrm{CI}$ : 1.99 to $2.76, \mathrm{p}<0.001 ; \mathrm{I}^{2}=36.5 \%, p_{\text {het }}=0.078$ ) or mGPS (HR: $1.69,95 \%$ CI: 1.49 to $\left.1.92, \mathrm{p}<0.001 ; \mathrm{I}^{2}=48.4 \%, p_{\text {het }}=0.022\right)$, and low level of LMR (HR: $1.37,95 \%$ CI: 1.14 to 1.65 , $\left.\mathrm{p}=0.001 ; \mathrm{I}^{2}=84.9 \%, p_{h e t}<0.001\right)$ and PNI (HR: $1.51,95 \%$ CI: 1.36 to $\left.1.68, \mathrm{p}<0.001 ; \mathrm{I}^{2}=45.8 \%, p_{\text {het }}=0.048\right)$.

Patients with an elevated NLR (HR: 1.21, 95\% CI: 1.04 to $\left.1.41, \mathrm{p}=0.011 ; \mathrm{I}^{2}=43.4 \%, p_{h e t}=0.089\right)$ and GPS (HR: 1.64, 95\% CI: 1.33 to $\left.1.94, \mathrm{p}<0.001 ; \mathrm{I}^{2}=45.5 \%, p_{\text {het }}=0.119\right)$ had a worse CSS (figure 3I-J) .

NLR (HR: $1.39,95 \%$ CI: 1.10 to $1.75, \mathrm{p}=0.005 ; \mathrm{I}^{2}=60.9 \%$, $p_{\text {het }}=0.018$ ), PLR (HR: $1.30,95 \%$ CI: 1.12 to $1.51, \mathrm{p}<0.001$; $\left.\mathrm{I}^{2}=33.0 \%, p_{h e t}=0.202\right)$ and GPS (HR: 2.44, 95\% CI: 1.28 to $\left.4.66, \mathrm{p}<0.007 ; \mathrm{I}^{2}=57.5 \%, p_{h e t}=0.052\right)$ were negatively correlated with DFS. No significant association was found for LMR (HR: 1.08, 95\% CI: 0.85 to $1.38, \mathrm{p}=0.522$; $\left.\mathrm{I}^{2}=79.8 \%, p_{h e t}<0.001\right)$ (figure $\left.3 \mathrm{~K}-\mathrm{N}\right)$.

\section{Subgroup analysis and meta-regression}

Subgroup analysis and meta-regression were further conducted according to the cut-off value, sample size, follow-up time, sex, age, clinical stage and region (online additional file 3 ). The heterogeneity of OS studies was relatively low except LMR $\left(I^{2}=84.9 \%\right)$ and NLR $\left(I^{2}=61.7 \%\right)$. The pooled HR was significantly different between studies with more or less than 280 patients, indicating that the sample size may be the source of heterogeneity for LMR. Similarly, we found the source of heterogeneity for other indicators. The follow-up time may be the source of heterogeneity for PLR ( $p=0.004)$ and GPS $(p=0.027)$. The sample size may be the source of heterogeneity for SII $(p=0.047)$ and mGPS $(p=0.014)$. The sex ratio may be the source of heterogeneity for CAR $(p=0.045)$. In DFS analysis, we found that cut-off value and region may be the source of high heterogeneity of LMR $(p=0.034)$ and NLR ( $\mathrm{p}=0.018)$, respectively.

\section{Publication bias}

Begg and Egger's tests were applied to estimate the publication bias. As shown in online additional file 3, no significant publication bias was observed.

\section{Sensitivity analysis}

We performed a sensitivity analysis by excluding one study each time. As shown in online additional 4, the results were not substantially changed, demonstrating the reliability and stability of the current meta-analysis.

\section{Pooled sensitivity, specificity, DOR, and AUC of indicators}

We further extracted TP, FP, FN and TN from each study (online additional file 2) to calculate the pooled accuracy of each indicator for a 5-year OS. There were 11 studies for NLR, 11 studies for PLR, 7 studies for LMR, 6 studies for CAR, 6 studies for SII, 7 studies for PNI, 6 studies for GPS and 5 studies for mGPS.

\section{Threshold effect}

The Spearman correlation coefficient ( $p$ value) for NLR, PLR, LMR, PNI, SII, CAR, GPS and mGPS was 0.56 


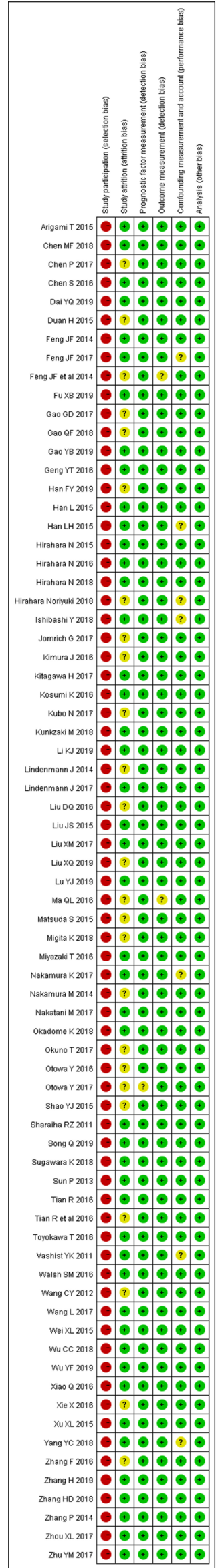

Figure 2 Risk of bias and applicability concerns.

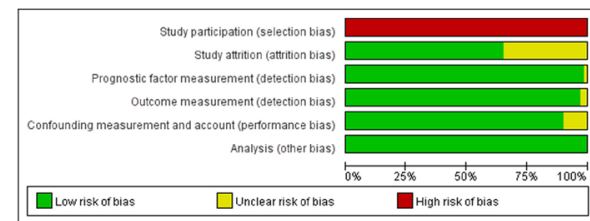




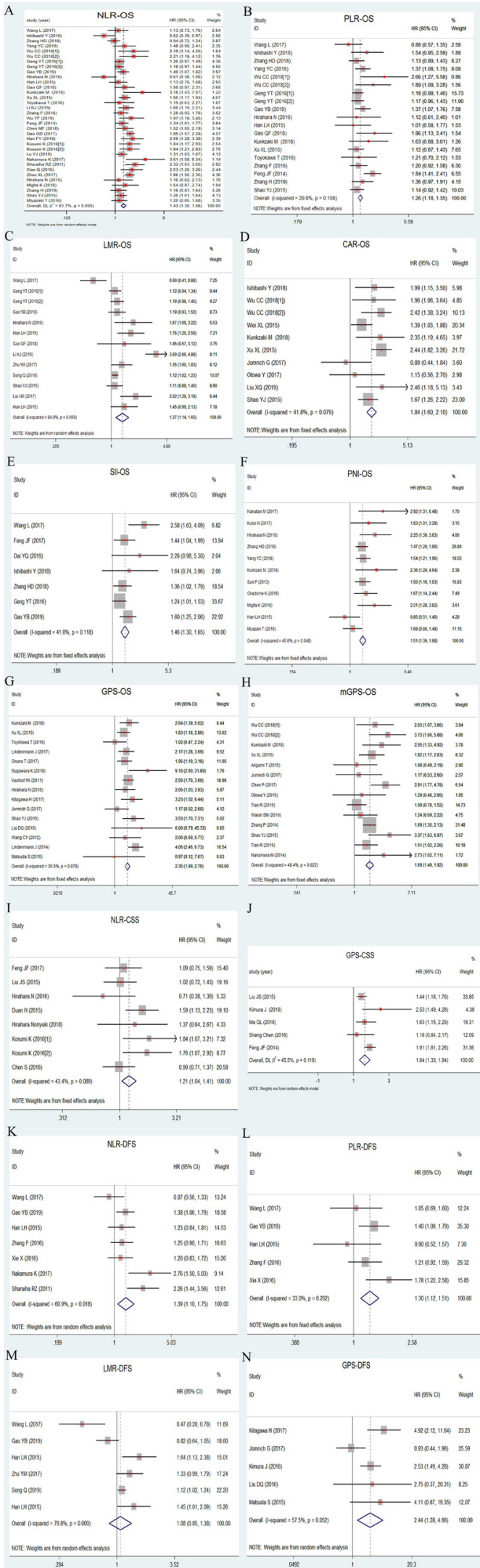

Figure 3 Forest plot of HR for 5- year OS, DFS and CSS in patients with oesophageal cancer. (A) NLR-OS; (B) PLR-OS; (C) LMR-OS; (D) CAR-OS; (E) SII-OS; (F) PNI-OS; (G) GPS-OS; (H) mGPS-OS; (I) NLR-CSS; (J) GPS-CSS; (K) NLR-DFS; (L) PLRDFS; (M) LMR-DFS; (N) GPS-DFS. CAR, C reactive protein-to-albumin ratio; CSS, cancer-specific survival; DFS, disease-free survival; GPS, Glasgow Prognostic Score; LMR, lymphocyte-to-monocyte ratio; mGPS, modified Glasgow Prognostic Score; NLR, neutrophil-to-lymphocyte ratio; OS, overall survival; PLR, platelet-to-lymphocyte ratio; PNI, prognostic nutritional index; SII, systemic inflammation index. 
(0.07), 0.59 (0.06), 0.57 (0.18), 0.75 (0.05), 0.77 (0.07), $0.20(0.70), 0.77(0.07)$ and $-0.10(0.87)$, respectively, indicating no significant threshold effect.

\section{Forest plot and subgroup analysis}

Forest plots of sensitivity and specificity are shown in figure 4 . SII had the highest pooled sensitivity $(0.61,95 \%$ CI: 0.48 to 0.73$)$, while CAR $(0.84,95 \%$ CI: 0.71 to 0.91$)$ had the highest pooled specificity. The $\mathrm{I}^{2}$ of the sensitivity and specificity of these prognostic indicators were relatively high (around 70\%-90\%) except GPS (sensitivity: $43.76 \%$; specificity: $6.96 \%$ ). We further conducted a subgroup analysis and meta-regression (online additional file 5). For SII, the sensitivity of studies with a cut-off level $\geq 410 \mathrm{ng} / \mathrm{mL}(0.47,95 \%$ CI: 0.37 to 0.57$)$ was significantly lower than the studies with a cut-off level $<410 \mathrm{ng} /$ $\mathrm{mL}(0.73,95 \%$ CI: 0.66 to 0.81$)$, while studies with a cutoff level $\geq 410 \mathrm{ng} / \mathrm{mL}(0.76,95 \%$ CI: 0.72 to 0.81$)$ had a significantly higher specificity than studies with a cut-off level $<410 \mathrm{ng} / \mathrm{mL}(0.42,95 \%$ CI: 0.38 to 0.47$)$. Therefore the cut-off value may be the source of heterogeneity in both sensitivity and specificity of SII. Similarly, we found that sample size may be the source of sensitivity for mGPS $(\mathrm{p}<0.001)$, PLR $(\mathrm{p}=0.02)$, GPS $(\mathrm{p}=0.03)$, CAR $(\mathrm{p}=0.04)$ and LMR $(\mathrm{p}=0.04)$, and the source of heterogeneity in the specificity of NLR $(p=0.03)$ and GPS $(p<0.001)$. Additionally, the study area may be the source of heterogeneity in the specificity of mGPS ( $p=0.01)$. Also, age and clinical stage may be the source of heterogeneity in specificity for PLR $(p<0.001)$ and PNI $(p=0.01)$, respectively. However, we failed to find the source of heterogeneity for the sensitivity of NLR or PNI and the specificity of CAR or LMR.

\section{Comparison of AUC}

Figure 5 shows the summarised receiver-operating characteristic curves of eight indicators. We found that the scope of pooled AUC of CAR $(0.72,95 \%$ CI: 0.68 to $0.75)$ and mGPS (0.75, 95\% CI: 0.71 to 0.78$)$ surpassed other indicators except GPS $(0.67,95 \%$ CI: 0.63 to 0.71$)$. We further compared CAR, mGPS and GPS by $z$ test. The pooled AUC of CAR or mGPS was larger than GPS $(\mathrm{p}=0.033 ; \mathrm{p}=0.002)$, but there was no significant difference between CAR and mGPS (online additional file 6).

\section{Publication bias and sensitivity analysis}

Only PNI ( $\mathrm{p}=0.03)$ and mGPS $(\mathrm{p}=0.02)$ had a significant publication bias (online additional file 7 ). The sensitivity analysis of combined DOR showed a robust finding (online additional file 4 and figure 2).

\section{DISCUSSION}

In this meta-analysis, we summarised data from 72 studies and estimated the predictive ability of inflammation and nutrition-based indicators in oesophageal cancer. In general, these indicators showed an excellent ability to predict the OS, DFS and CSS of patients with oesophageal cancer. The pretreatment level of CAR and mGPS showed an outstanding prediction value for 5-year OS than other indicators.

Inflammation plays an essential role in the development and progression of various malignant tumours. ${ }^{14} \mathrm{In}$ addition, nutritional status is closely related to carcinogenesis, cancer growth, tumour progression and tumour prognosis. ${ }^{15}$ The peripheral blood cell analysis is a good choice for establishing a prognostic prediction model based on inflammatory and nutrition-related indicators due to its convenience, repeatability and low cost. ${ }^{16}$ Previous studies have systematically reviewed the role of some inflammation and nutrition-based indicators in the prognosis of oesophageal cancer, most of which focused on ESCC. Yang et $a l^{17}$ investigated the relationship between NLR and oesophageal cancer by summarising six studies involving 1633 patients. Sun and Zhang ${ }^{5}$ reviewed 26 studies to explore the NLR, PLR and LMR in the OS, CSS and DFS in ESCC. Li et $l^{18}$ reviewed nine observational studies and showed that a low PNI score was significantly correlated with a poor OS of oesophageal cancer and recurrence-free survival of ESCC. Liu et al collected eight observational studies and showed that high CAR was related to a worse OS. ${ }^{19}$ Although previous meta-analyses have reported the prognostic value of these indicators, this is the first study to comprehensively estimate the popular inflammatory and nutrition-related markers in OS, DFS and CSS of oesophageal cancer. Moreover, this is the first systematic review to summarise the sensitivity and specificity and compare the AUC of these predictors in the 5-year OS of oesophageal cancer.

In this review, we observed that the AUC of CAR and mGPS was significantly higher than NLR, PLR, SII, PNI, LMR and GPS, indicating their outstanding predictive value in oesophageal cancer. CAR and mGPS are calculated based on the level of CRP and albumin. CRP is a kind of acute reactive protein synthesised by liver cells or cancer cells, which can produce an attractive environment for tumour growth, induce DNA damage, promote angiogenesis and favour neoplastic spread and metastasis, revealing levels of inflammation in the body. ${ }^{20} 21$ Albumin reflects the malnutrition status of the host, triggers malignant transformation and tumour progression or even causes cachexia. ${ }^{22}$ Combining the two indicators can reveal a patient's inflammatory status and nutritional status, which can effectively predict prognosis. These may explain the prominent prognostic role of CAR and mGPS. Additionally, some prospective studies have revealed the better predictive power of CAR and mGPS in other types of cancer. For example, it was reported that the CAR had a better predictive performance for hepatocellular carcinoma and colorectal cancer than NLR, PLR or CRP alone. ${ }^{23}$ Other studies demonstrated that mGPS was an independent marker of poor prognosis for patients with SCC and superior to NLR, PLR and PNI. ${ }^{9}$

Although the TNM staging system is well known as a predictive clinical parameter in terms of guiding treatment and clinical prognosis, the survival outcomes for patients with oesophageal cancer with the same TNM 
A

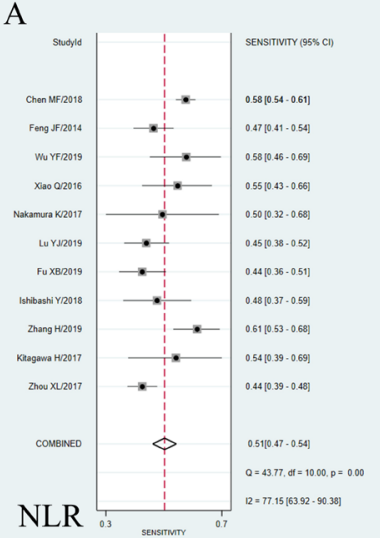

$\mathrm{C}$

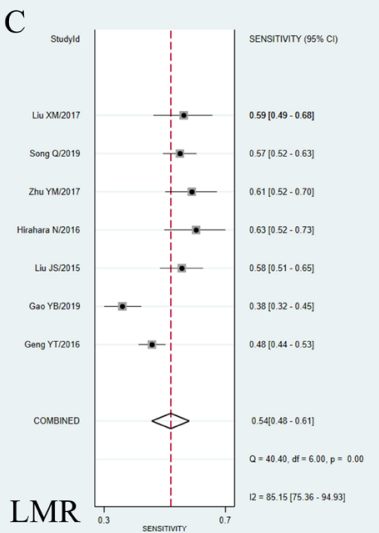

E

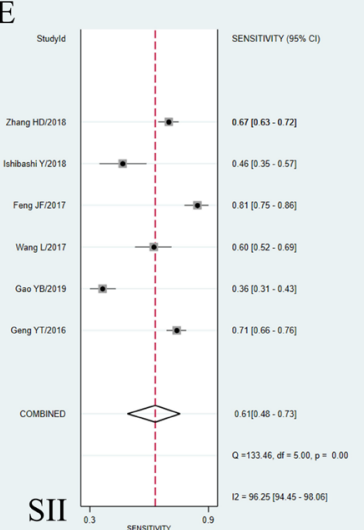

G

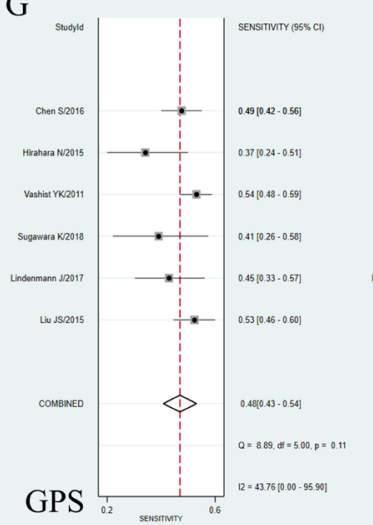

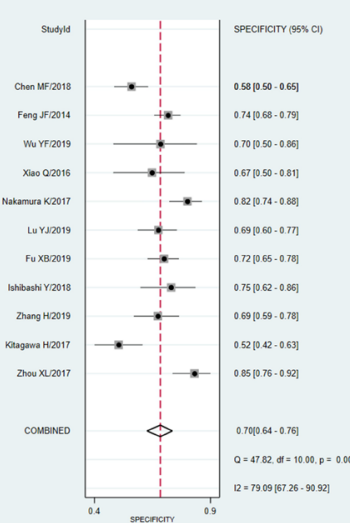
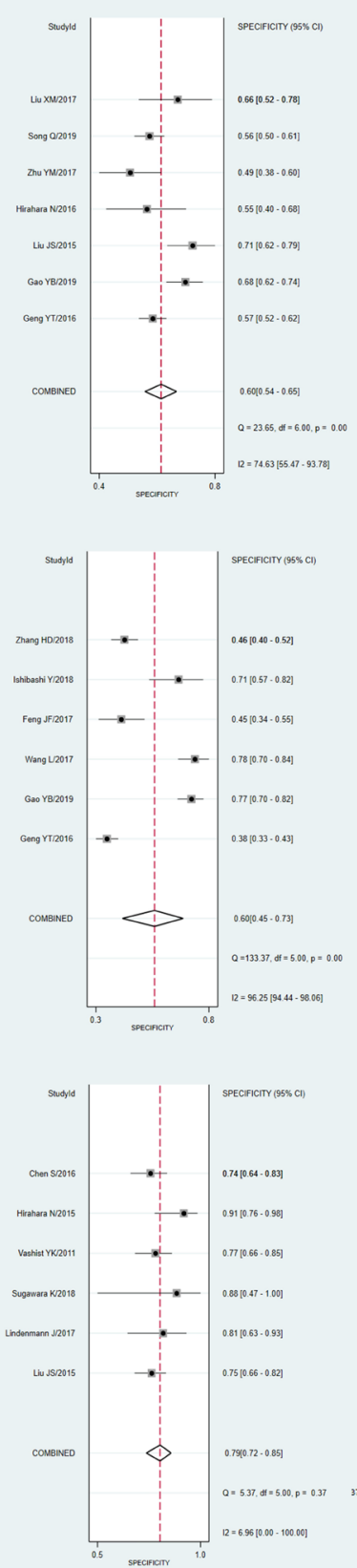

B
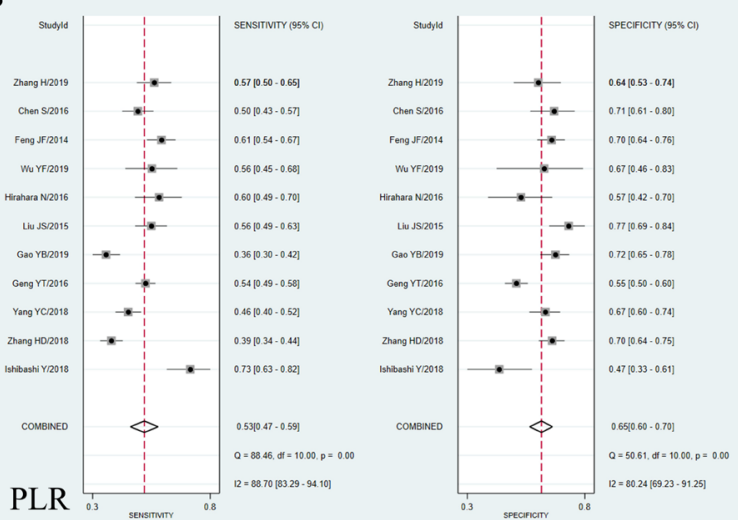

D

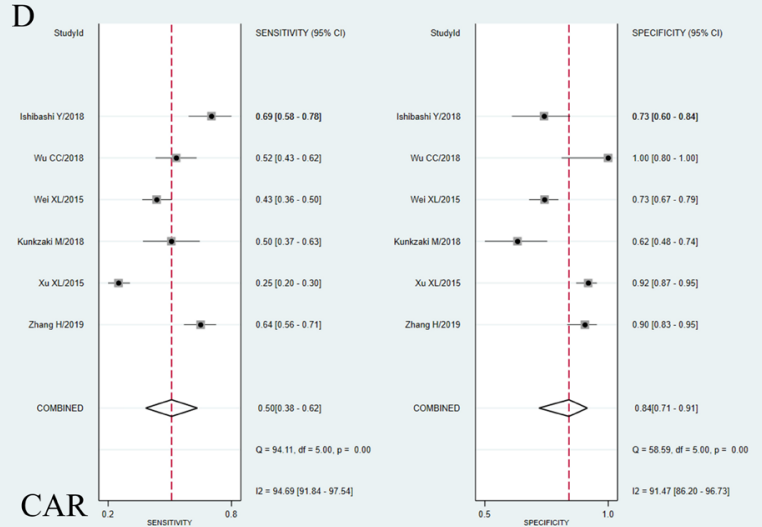

$\mathrm{F}$

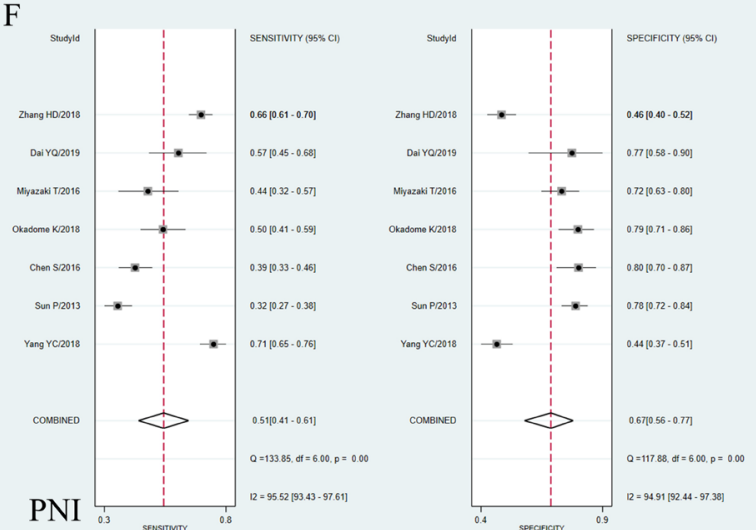

$\mathrm{H}$

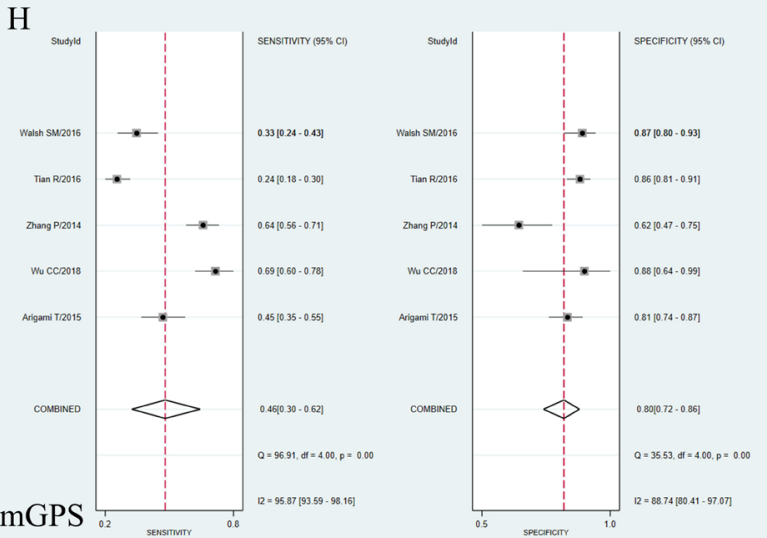



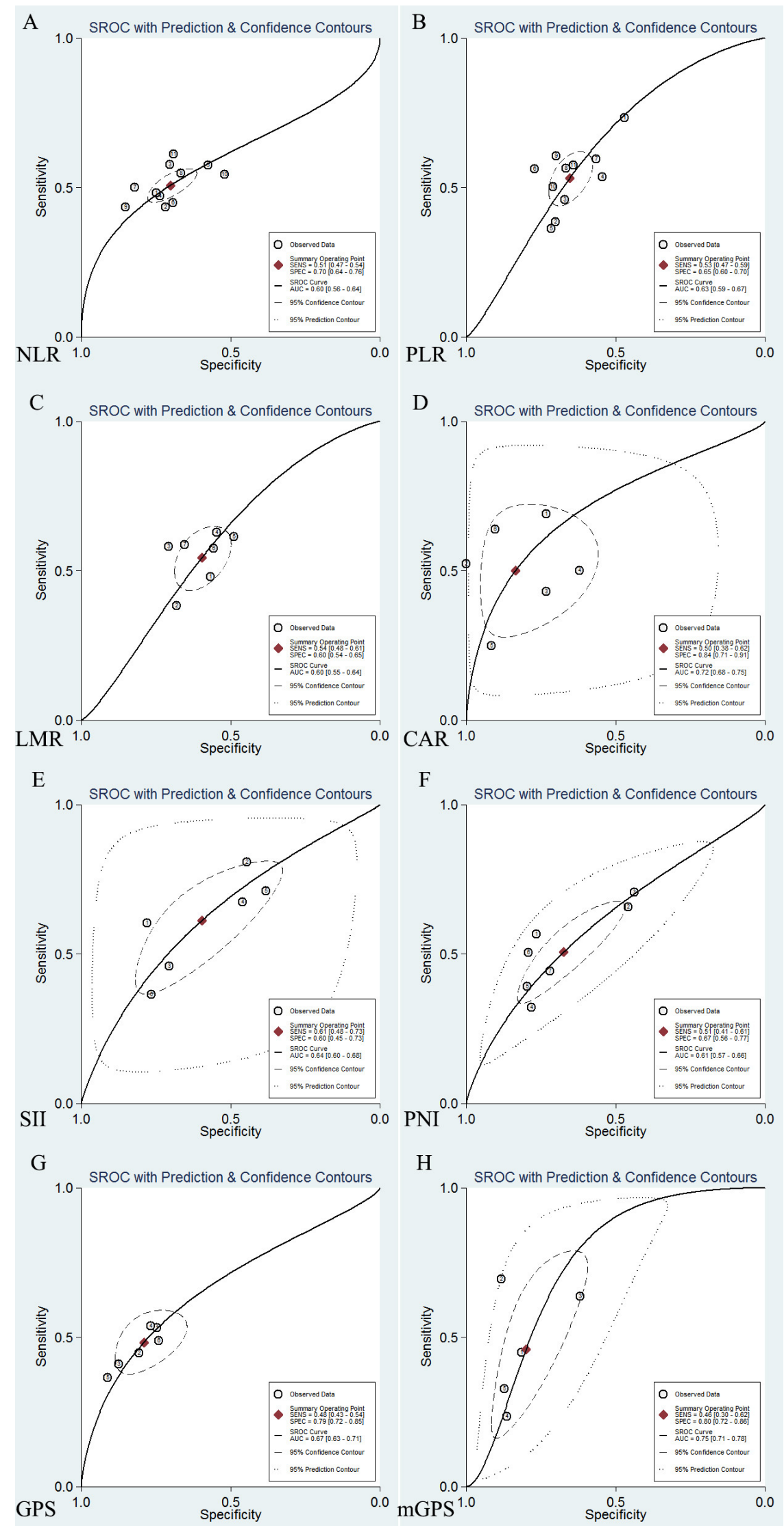

Figure 5 SROC curves of 5-year overall survival. (A) NLR; (B) PLR; (C) LMR; (D) CAR; (E) SII; (F) PNI; (G) GPS; (H) mGPS; AUC, area under curve. CAR, C reactive protein-to-albumin ratio; GPS, Glasgow Prognostic Score; LMR, lymphocyte-to-monocyte ratio; mGPS, modified Glasgow Prognostic Score; NLR, neutrophil-to-lymphocyte ratio; PLR, platelet-to-lymphocyte ratio; PNI, prognostic nutritional index; SII, systemic inflammation index. SROC, summary receiver-operating characteristic. 
stage still vary widely. ${ }^{4}$ In addition, many patients with oesophageal cancer cannot undergo surgery to obtain pathological identification for various reasons. Thus their prognosis cannot be obtained. Moreover, multiple factors influence the prognosis of patients with oesophageal cancer, such as neoadjuvant therapy, psychological factors and behaviour and eating habits, which will change the postoperative pathological stage of the tumour and thus affect the evaluation of disease progression. ${ }^{24-26}$ Therefore, pathological diagnosis is not sufficient to accurately predict the prognosis of patients with oesophageal cancer. More readily available objective indicators with high specificity and sensitivity are needed to predict the prognosis of patients with cancer. Our results of this meta-analysis will help clinicians and patients to select appropriate indicators for prognosis prediction. In this way, patients can be classified, and appropriate treatment strategies and postoperative management methods can be selected, providing policymakers with ideas. Taken mGPS as an example, patients with oesophageal cancer with a score of 2 may have a high risk of prognosis, which may provide an effective way for clinicians to select highrisk patients with worse prognosis or severe adverse events before treatment and further timely adjust individualised treatment regimens and enhance postoperative rehabilitation. In addition, policymakers should develop policies to strengthen community guidance and management of such high-risk postoperative patients.

Malnutrition is closely related to carcinogenesis, cancer growth, tumour progression and tumour prognosis. ${ }^{27}$ The Global Leadership Initiative on Malnutrition (GLIM) standards integrate current best evidence and expert opinion on malnutrition to promote the prevention, identification and treatment of malnutrition in patients with cancer. ${ }^{28}$ Inflammation is one of the aetiological criteria in GLIM classification, and studies have demonstrated that the changes of GPS score, CRP and albumin are highly consistent with the GLIM criteria in identifying malnutrition in patients. ${ }^{29}$ Similar to this study, our findings confirm the value of mGPS and CAR in predicting the prognosis of oesophageal cancer. GLIM criteria are re-evaluated every 3-5 years based on new research. Our results may provide a basis for the optimisation of GLIM criteria. Additionally, previous studies have reported that the combination of PNI and GLIM criteria has significant advantages in predicting the incidence and survival rate of perioperative malnutrition. ${ }^{30}$ Our results show that the prognostic indicators we studied have high specificity but unsatisfactory sensitivity. More well-designed studies are needed to develop joint indicators to improve the sensitivity and specificity of prediction.

Some limitations should be acknowledged. First, some heterogeneity was not fully explained. This may be due to the fact that some factors that may affect survival were not included, such as living behaviour and eating habits, comorbidities, neoadjuvant therapy and psychological factors. ${ }^{1024}$ Second, the cut-off value of indicators varied between studies, affecting the pooled analysis results and induce unavoidable potential heterogeneity and bias. Therefore, a standard and uniform cut-off value need to be defined. Third, publication bias was detected in studies on PNI and mGPS. Papers that failed to get published due to negative or null results could not be identified in our literature search and thus were not included in the metaanalysis. This may overestimate the prognostic effect of PNI and mGPS. Therefore, more well-designed prospective studies with large samples are needed to verify our findings.

\section{CONCLUSION}

NLR, PLR, LMR, PNI, SII, CAR, GPS and mGPS are commonly used as clinical indicators to predict OS, DFS and CSS of oesophageal cancer, but with unsatisfactory sensitivity. Pretreatment CAR and mGPS showed outstanding prognostic values in 5-year OS for patients with oesophageal cancer. Future extensive prospective studies with rigorously designed methodologies are warranted to confirm our results.

Contributors YJ and DX were mainly responsible for data collection, data analysis, drafting and revision. JW participated in the topic design, work plan and paper revision. HS and BQ helped complete the data collection. DT, YJ and ZL involved in the data analysis and paper revision. YJ, DX and HS contributed equally to this paper. All authors finally approved the version to be published and agreed to be accountable for all aspects of the work.

Funding The present study was supported by the National Natural Science Foundation of China $(82173595,81673249)$, Postgraduate Research \& Practice Innovation Program of Jiangsu Province (KYCX20_1411) and Priority Academic Program Development of Jiangsu Higher Education Institutions (PAPD). The funding agencies had no role in the study design, data collection, analysis, decision to publish or preparation of the manuscript.

\section{Competing interests None declared.}

Patient consent for publication Not required.

Ethics approval All data were downloaded from the public database and followed the data access policies. This study was exempted from ethical review by the ethics committee of Nanjing Medical University. This study did not involve individual information, so there was no requirement for informed consent.

Provenance and peer review Not commissioned; externally peer reviewed.

Data availability statement Data are available in a public, open access repository. All data generated or analyzed during this study are included in this published article.

Supplemental material This content has been supplied by the author(s). It has not been vetted by BMJ Publishing Group Limited (BMJ) and may not have been peer-reviewed. Any opinions or recommendations discussed are solely those of the author(s) and are not endorsed by BMJ. BMJ disclaims all liability and responsibility arising from any reliance placed on the content. Where the content includes any translated material, BMJ does not warrant the accuracy and reliability of the translations (including but not limited to local regulations, clinical guidelines, terminology, drug names and drug dosages), and is not responsible for any error and/or omissions arising from translation and adaptation or otherwise.

Open access This is an open access article distributed in accordance with the Creative Commons Attribution Non Commercial (CC BY-NC 4.0) license, which permits others to distribute, remix, adapt, build upon this work non-commercially, and license their derivative works on different terms, provided the original work is properly cited, appropriate credit is given, any changes made indicated, and the use is non-commercial. See: http://creativecommons.org/licenses/by-nc/4.0/.

\section{ORCID iDs}

Yan Jiang http://orcid.org/0000-0001-7312-8708

Beibei Qiu http://orcid.org/0000-0002-7053-7503

Jianming Wang http://orcid.org/0000-0002-9151-284X 


\section{REFERENCES}

1 Arnold M, Abnet CC, Neale RE, et al. Global burden of 5 major types of gastrointestinal cancer. Gastroenterology 2020;159:335-49.

2 Sung H, Ferlay J, Siegel RL, et al. Global cancer statistics 2020: GLOBOCAN estimates of incidence and mortality worldwide for 36 cancers in 185 countries. CA Cancer J Clin 2021;71:209-49.

3 Huang F-L, Yu S-J. Esophageal cancer: risk factors, genetic association, and treatment. Asian J Surg 2018;41:210-5.

4 Gong YB, Zhu Z, Wang X, et al. [Influence of different biological behaviors on prognosis of patients with advanced gastric cancer at the same TNM stage]. Zhonghua Wei Chang Wai Ke Za Zhi 2020;23:953-62.

5 Sun Y, Zhang L. The clinical use of pretreatment NLR, PLR, and LMR in patients with esophageal squamous cell carcinoma: evidence from a meta-analysis. Cancer Manag Res 2018;10:6167-79.

6 Zhang $\mathrm{H}$, Shang X, Ren $\mathrm{P}$, et al. The predictive value of a preoperative systemic immune-inflammation index and prognostic nutritional index in patients with esophageal squamous cell carcinoma. J Cell Physiol 2019;234:1794-802.

7 Fu X, Li T, Dai Y, et al. Preoperative systemic inflammation score (SIS) is superior to neutrophil to lymphocyte ratio (NLR) as a predicting indicator in patients with esophageal squamous cell carcinoma. BMC Cancer 2019;19:721.

8 Okadome K, Baba Y, Yagi T, et al. Prognostic nutritional index, tumorinfiltrating lymphocytes, and prognosis in patients with esophageal cancer. Ann Surg 2020;271:693-700.

9 Fan H, Shao Z-Y, Xiao Y-Y, et al. Comparison of the glasgow prognostic score (GPs) and the modified glasgow prognostic score (mGPS) in evaluating the prognosis of patients with operable and inoperable non-small cell lung cancer. J Cancer Res Clin Oncol 2016;142:1285-97.

10 Akgun E, Ozkok S, Tekin M, et al. The effects of chemoradiotherapy on recurrence and survival in locally advanced rectal cancers with curative total mesorectal excision: a prospective, nonrandomized study. World J Surg Oncol 2017;15:205.

11 Whiting PF, Rutjes AWS, Westwood ME, et al. QUADAS-2: a revised tool for the quality assessment of diagnostic accuracy studies. Ann Intern Med 2011;155:529-36.

12 Zamora J, Abraira V, Muriel A, et al. Meta-disc: a software for metaanalysis of test accuracy data. BMC Med Res Methodol 2006;6:31.

13 Moses LE, Shapiro D, Littenberg B. Combining independent studies of a diagnostic test into a summary ROC curve: dataanalytic approaches and some additional considerations. Stat Med 1993;12:1293-316.

14 Ostan R, Lanzarini C, Pini E, et al. Inflammaging and cancer: a challenge for the mediterranean diet. Nutrients 2015;7:2589-621.

15 Steenhagen E, van Vulpen JK, van Hillegersberg R, et al. Nutrition in peri-operative esophageal cancer management. Expert Rev Gastroenterol Hepatol 2017;11:663-72.
16 Wu Y, Ye S, Goswami S, et al. Clinical significance of peripheral blood and tumor tissue lymphocyte subsets in cervical cancer patients. BMC Cancer 2020;20:173.

17 Yang $\mathrm{Y}, \mathrm{Xu} \mathrm{H}$, Zhou L, et al. Platelet to lymphocyte ratio is a predictive marker of prognosis and therapeutic effect of postoperative chemotherapy in non-metastatic esophageal squamous cell carcinoma. Clin Chim Acta 2018;479:160-5.

18 Li P, Wang X, Lai Y, et al. The prognostic value of pre-treatment prognostic nutritional index in esophageal squamous cell carcinoma: a meta-analysis. Medicine 2019;98:e15280.

19 Liu Z, Shi H, Chen L. Prognostic role of pre-treatment C-reactive protein/albumin ratio in esophageal cancer: a meta-analysis. $B M C$ Cancer 2019;19:1161.

20 Aiolfi A, Asti E, Rausa E, et al. Use of C-reactive protein for the early prediction of anastomotic leak after esophagectomy: systematic review and Bayesian meta-analysis. PLoS One 2018;13:e0209272.

21 Morris-Stiff G, Gomez D, Prasad KR. C-reactive protein in liver cancer surgery. Eur J Surg Oncol 2008;34:727-9.

22 Mantzorou M, Koutelidakis A, Theocharis S, et al. Clinical value of nutritional status in cancer: what is its impact and how it affects disease progression and prognosis? Nutr Cancer 2017;69:1151-76.

$23 \mathrm{Ni}$ X-C, Yi Y, Fu Y-P, et al. Prognostic value of the modified Glasgow prognostic score in patients undergoing radical surgery for hepatocellular carcinoma. Medicine 2015;94:e1486.

24 Taira N, Iwata H, Hasegawa Y, et al. Health-related quality of life and psychological distress during neoadjuvant endocrine therapy with letrozole to determine endocrine responsiveness in postmenopausal breast cancer. Breast Cancer Res Treat 2014;145:155-64.

25 Pierce JP, Natarajan L, Caan BJ, et al. Influence of a diet very high in vegetables, fruit, and fiber and low in fat on prognosis following treatment for breast cancer: the women's healthy eating and living (WHEL) randomized trial. JAMA 2007;298:289-98.

26 Ohashi S, Miyamoto Shin'ichi, Kikuchi O, et al. Recent advances from basic and clinical studies of esophageal squamous cell carcinoma. Gastroenterology 2015;149:1700-15.

27 Marian M, August DA. Prevalence of malnutrition and current use of nutrition support in cancer patient study. JPEN J Parenter Enteral Nutr 2014;38:163-5.

28 Jensen GL, Cederholm T, Correia MITD, et al. GLIM criteria for the diagnosis of malnutrition: a consensus report from the global clinical nutrition community. JPEN J Parenter Enteral Nutr 2019;43:32-40.

29 Contreras-Bolívar V, Sánchez-Torralvo FJ, Ruiz-Vico M, et al. GLIM criteria using hand grip strength adequately predict six-month mortality in cancer inpatients. Nutrients 2019;11:2043.

30 Wang P, Chen X, Liu Q, et al. Good performance of the global leadership initiative on malnutrition criteria for diagnosing and classifying malnutrition in people with esophageal cancer undergoing esophagectomy. Nutrition 2021;91-92:111420-2. 